Condorcet Efficiency: A Preference for Indifference

\author{
William V. Gehrlein \\ Department of Business Administration \\ University of Delaware \\ Newark, DE 19716 \\ USA
}

Fabrice Valognes

GREBE

Department of Economics

The University of Namur

B-5000 Namur

BELGIUM

JEL Classification: D72

Key Words: Condorcet Efficiency, Probability, Voting Rules 
Condorcet Efficiency: A Preference for Indifference

\begin{abstract}
The Condorcet winner in an election is the candidate who would be able to defeat all other candidates in a series of pairwise elections. The Condorcet efficiency of a voting procedure is the conditional probability that it will elect the Condorcet winner, given that a Condorcet winner exists. The study considers the Condorcet efficiency of weighted scoring rules (WSR's) on three candidates for large electorates when voter indifference between candidates is allowed. It is shown that increasing the proportion of voters who have partial indifference will increase the probability that a Condorcet winner exists, and will also increase the Condorcet efficiency of all WSR's. The same observation is observed when the proportion of voters with complete preferences on candidates is reduced. Borda Rule is shown to be the WSR with maximum Condorcet efficiency over a broad range of assumptions related to voter preferences. The result of forcing voters to completely rank all candidates, by randomly breaking ties on candidates that are viewed as indifferent, leads to a reduction in the probability that a Condorcet winner exists and to a reduction in the Condorcet efficiency of all WSR's.
\end{abstract}




\section{Condorcet Efficiency: A Preference for Indifference}

\section{Introduction}

When two candidates are being considered in an election, it is difficult to argue against the basic notion that majority rule should be implemented to determine the winner. However, many different criteria have been established to determine who the winner should be when more than two candidates are being considered. One criterion that has received a great deal of attention is attributed to Condorcet (1785). For a three candidate election, the Condorcet winner would be the candidate who would be able to defeat each of the other two candidates by majority rule in pairwise elections. It is well known that a Condorcet winner does not always exist, but the Condorcet winner would seem to be a good candidate for selection when one does exist. The Condorcet efficiency of a voting rule is defined as the conditional probability that it elects the Condorcet winner, given that one exists. An extensive survey of research on the probability that a Condorcet winner exists and the Condorcet efficiency of voting rules is given in Gehrlein (1997).

Most of the research to date on the probability that there is a Condorcet winner and on Condorcet efficiency has focused on the situation in which all voters have complete preference rankings on the candidates, with no indifference allowed.

Exceptions to this have dealt with the probability that there is a Condorcet winner when indifference is allowed [Van Deemen (1999), Lepelley and Martin (1998), Fishburn and Gehrlein (1980), Jones, Radcliff, Taber and Timpone (1995)]. The purpose of the current study is to consider the impact that voter indifference on candidates will have on the Condorcet efficiency of voting rules. Attention is restricted to the case of three candidate elections in the limiting case of a large electorate. The probability that voters will have given preferences on candidates is assumed to follow an extension of the widely used impartial culture condition. 


\section{Impartial Culture with Indifference}

Consider three candidates $[\mathrm{A}, \mathrm{B}, \mathrm{C}]$ in an election. Let $\mathrm{A} \succ \mathrm{B}$ denote the situation in which a voter prefers candidate $A$ to candidate $B$. Then, $A \sim B$ denotes the situation of voter indifference between candidate $\mathrm{A}$ and candidate $\mathrm{B}$. There are six possible complete preference rankings on the candidates that might represent the preferences of a voter when indifference between candidates is not allowed:

$\begin{array}{llllll}\mathrm{A} & \mathrm{A} & \mathrm{B} & \mathrm{C} & \mathrm{B} & \mathrm{C} \\ \mathrm{B} & \mathrm{C} & \mathrm{A} & \mathrm{A} & \mathrm{C} & \mathrm{B} \\ \mathrm{C} & \mathrm{B} & \mathrm{C} & \mathrm{B} & \mathrm{A} & \mathrm{A} \\ \mathrm{p}_{1} & \mathrm{p}_{2} & \mathrm{p}_{3} & \mathrm{p}_{4} & \mathrm{p}_{5} & \mathrm{p}_{6}\end{array}$

where $p_{i}$ is the probability that a randomly selected voter has the associated preference ranking on candidates. The condition of impartial culture (IC) is a commonly used assumption for studies that do not allow indifference between candidates in voter preference structures. With IC, it is assumed that $p_{i}=1 / 6$ for each preference ranking with $1 \leq \mathrm{i} \leq 6$, and that all voters arrive at their preferences independently.

We wish to extend the notion of IC, to apply it to the case that allows for indifference between candidates in the preferences of voters. In general, we shall only require that voter preference structures be represented by weak orders. We let the voters with complete preference rankings, like those above, represent a class of voters. A second class of voters will have weak ordered preferences with partial indifference. An example of partial indifference would be a voter who has $\mathrm{A} \sim \mathrm{B}$ but feels that both $\mathrm{A} \succ \mathrm{C}$ and that $\mathrm{B} \succ \mathrm{C}$. There are six different weak ordered preference structures that represent partial indifference:

$\begin{array}{cccccc}\mathrm{A} \sim \mathrm{B} & \mathrm{A} \sim \mathrm{C} & \mathrm{B} \sim \mathrm{C} & \mathrm{A} & \mathrm{B} & \mathrm{C} \\ \mathrm{C} & \mathrm{B} & \mathrm{A} & \mathrm{B} \sim \mathrm{C} & \mathrm{A} \sim \mathrm{C} & \mathrm{A} \sim \mathrm{B} \\ \mathrm{p}_{7} & \mathrm{p}_{8} & \mathrm{p} 9 & \mathrm{p}_{10} & \mathrm{p}_{11} & \mathrm{p}_{12}\end{array}$

The third class of voters will have preferences that represent complete indifference, with $\mathrm{A} \sim \mathrm{B}, \mathrm{A} \sim \mathrm{C}$ and $\mathrm{B} \sim \mathrm{C}$. Let $\mathrm{p}_{13}$ denote the probability that a voter has preferences represented by complete indifference. 
Let $\mathrm{k}_{1}$ denote the probability that a randomly selected voter is among the class of voters with complete preference rankings. Similarly, $\mathrm{k}_{2}$ denotes the probability for voters with partial indifference, and $\mathrm{k}_{3}$ denotes the probability for voters with complete indifference on candidates. We then have $\mathrm{k}_{1}+\mathrm{k}_{2}+\mathrm{k}_{3}=1$. The impartial weak order culture condition (IWOC) then assumes that all preference structures within a class of voter preference types are equally likely to be observed. With the voter preferences that we defined above: $p_{i}=k_{1} / 6$ for $i=1,2,3,4,5,6 ; p_{i}=k_{2} / 6$ for $i=7,8,9,10,11,12$; and $k_{3}=p_{13}$. Let the vector $\mathbf{k}=\left(\mathrm{k}_{1}, \mathrm{k}_{2}, \mathrm{k}_{3}\right)$.

Fishburn and Gehrlein (1980) considered the assumption of IWOC for the case of three candidates in the limiting case for voters to develop a representation, $\mathrm{P}_{\mathrm{Con}}^{\infty}$ (IWOC), for the probability that a Condorcet winner exists, with a given k. The resulting representation is given by

$$
\mathrm{P}_{\text {Con }}^{\infty}(\mathrm{IWOC})=\frac{3}{4}+\frac{3}{2 \pi} \operatorname{Sin}^{-1}\left(\frac{\mathrm{k}_{1}+\mathrm{k}_{2}}{3 \mathrm{k}_{1}+2 \mathrm{k}_{2}}\right)
$$

For the special case $\mathrm{k}_{1}=1\left(\mathrm{k}_{2}=0\right)$, this reduces to the original case of IC, and a result due to Guilbaud (1952) is obtained for $\mathrm{P}_{\mathrm{Con}}^{\infty}$ (IC) as a special case. Obviously, $\mathrm{k}_{1}+\mathrm{k}_{2}>0$ in this representation since the situation of complete indifference for all voters $\left(\mathrm{k}_{3}=1\right)$ could not lead to the existence of a Condorcet winner, and the case of $\mathrm{k}_{1}=\mathrm{k}_{2}=0$ is accordingly undefined in the representation.

Some interesting results that will be used later follow directly from the stated representation for $\mathrm{P}_{\mathrm{Con}}^{\infty}(\mathrm{IWOC})$. These results were first suggested by Inada (1964).

Lemma 1. For any fixed $\mathrm{k}_{1}>0, \mathrm{P}_{\mathrm{Con}}^{\infty}(\mathrm{IWOC})$ increases as $\mathrm{k}_{2}$ increases. For $\mathrm{k}_{1}=0$, $\mathrm{P}_{\mathrm{Con}}^{\infty}($ IWOC $)=1$ with any $\mathrm{k}_{2}>0$.

Proof: The first part is obtained directly by differentiating $\mathrm{P}_{\text {Con }}^{\infty}$ (IWOC) with respect to $\mathrm{k}_{2}$, and the second part follows directly from the definition of $\mathrm{P}_{\mathrm{Con}}^{\infty}($ IWOC) with the fact that $\operatorname{Sin}^{-1}\left(\frac{1}{2}\right)=\frac{\pi}{6}$. 
Lemma 2. For any fixed $\mathrm{k}_{2}>0, \mathrm{P}_{\mathrm{Con}}^{\infty}(\mathrm{IWOC})$ decreases as $\mathrm{k}_{1}$ increases.

Proof: The proof is obtained directly by differentiating $\mathrm{P}_{\mathrm{Con}}^{\infty}$ (IWOC) with respect to $\mathrm{k}_{1}$.

QED

As a result of these lemmas, we observe two major trends. First, as voters' preferences shift from complete indifference to partial indifference, there is an increase in the probability that a Condorcet winner exists. The second observation is somewhat more surprising, in that we see a decrease in the probability that there is a Condorcet winner as voters' preferences shift from complete indifference to complete preference rankings. A simple extension is that there is a decrease in the probability that there is a Condorcet winner as voters' preferences shift from partial indifference to complete preference rankings.

Having considered the probability that a Condorcet winner exists with IWOC and the results that were observed, we turn our attention to the probability that various common election procedures select the Condorcet winner when there is one. It will be of particular interest to determine the effect that the existence of partial indifference will have on the Condorcet efficiency of weighted scoring rules.

\section{Condorcet Efficiency with IWOC}

The Condorcet efficiency of a voting rule has been defined as the conditional probability that the particular voting rule elects the Condorcet winner, given that a Condorcet winner exists. We consider the family of voting rules that can be represented by weighted scoring rules. A weighted scoring rule (WSR) for three candidates assigns weights of $1, \lambda$, and 0 points respectively to each voter's first, second, and third ranked candidate according to their preferences on the candidates. The winner is then selected as the candidate who receives the most total points over all voters. The specific case with $\lambda=0$ represents the commonly used plurality rule, and the case of $\lambda=1$ is negative plurality rule. 
A WSR can be applied directly to voters with complete preference rankings, but some modification must be made when indifference exists. To assure that all voters have the same overall input to the election, we require that each voter has a total of $1+\lambda$ points to distribute to candidates. Suppose that a voter has preferences with partial indifference such that $\mathrm{A} \succ \mathrm{B}, \mathrm{A} \succ \mathrm{C}$, and $\mathrm{B} \sim \mathrm{C}$. For any complete ranking that includes these preferences, A would always be ranked first, so we will give A a weight of one with the WSR. Candidates $\mathrm{B}$ and $\mathrm{C}$ would receive either $\lambda$ or 0 points in a complete ranking that includes these preferences, so we will give both $B$ and $C$ an average weight of $\lambda / 2$ with the WSR. Using the same basic notion, with voters having partial indifference of the form $\mathrm{B} \succ \mathrm{A}, \mathrm{C} \succ \mathrm{A}$, and $\mathrm{B} \sim \mathrm{C}$; we assign zero points to $\mathrm{A}$ and $(1+\lambda) / 2$ points to each of B and C. For voters with complete indifference, each candidate will be given $(1+\lambda) / 3$ points by the WSR.

To find the Condorcet efficiency of WSR with weight $\lambda$, which we denote as Rule $\lambda$, we need a representation for the joint probability, $\mathrm{J}(\lambda, \mathbf{k}, \mathrm{IWOC})$, that some candidate, say candidate $\mathrm{A}$, is both the Condorcet winner and is elected by Rule $\lambda$ under IWOC. To develop a representation for $\mathrm{J}(\lambda, \mathbf{k}, \mathrm{IWOC})$, we begin by defining four discrete variables, which have values that are determined by the $p_{i}$ probabilities that are associated with voter preference structures:

$$
\begin{aligned}
& \mathrm{X}_{1}=1: \mathrm{p}_{1}+\mathrm{p}_{2}+\mathrm{p}_{4}+\mathrm{p}_{8}+\mathrm{p}_{10} \\
& -1: \mathrm{p}_{3}+\mathrm{p}_{5}+\mathrm{p}_{6}+\mathrm{p}_{9}+\mathrm{p}_{11} \\
& \mathrm{X}_{3}=(1-\lambda): \mathrm{p}_{1} \\
& 1: p_{2} \\
& (\lambda-1): p_{3} \\
& \lambda: p_{4} \\
& -1: p_{5} \\
& -\lambda: p_{6} \\
& (1+\lambda) / 2: p_{8} \\
& -(1+\lambda) / 2: p_{9} \\
& (2-\lambda) / 2: \mathrm{p}_{10} \\
& (\lambda-2) / 2: p_{11}
\end{aligned}
$$

$$
\begin{aligned}
& \mathrm{X}_{2}=1: \mathrm{p}_{1}+\mathrm{p}_{2}+\mathrm{p}_{3}+\mathrm{p}_{7}+\mathrm{p}_{10} \\
& -1: \mathrm{p}_{4}+\mathrm{p}_{5}+\mathrm{p}_{6}+\mathrm{p}_{9}+\mathrm{p}_{12} \\
& \mathrm{X}_{4}=(1-\lambda): \mathrm{p}_{2} \\
& 1: p_{1} \\
& (\lambda-1): \mathrm{p}_{4} \\
& \lambda: \mathrm{p}_{3} \\
& -1: p_{6} \\
& -\lambda \quad: p_{5} \\
& (1+\lambda) / 2: p_{7} \\
& -(1+\lambda) / 2: \mathrm{p}_{9} \\
& (2-\lambda) / 2: \mathrm{p}_{10} \\
& (\lambda-2) / 2: p_{12}
\end{aligned}
$$

With these definitions of the $\mathrm{X}_{\mathrm{i}}$ 's, we note that A will be ranked over (under) B in a voter's preference structure when $X_{1}=+1(-1)$. Similarly, A will be ranked over 
(under) $\mathrm{C}$ in a voter's preference structure when $\mathrm{X}_{2}=+1(-1)$. The value of $\mathrm{X}_{3}\left(\mathrm{X}_{4}\right)$ represents the difference in weights that Rule $\lambda$ assigns to candidates $A$ and $B(C)$. If we have a total of $n$ voters and let $\bar{X}_{i}$ denote the average value of $X_{i}$ over the $n$ voters, it follows from the definitions that $\mathrm{J}(\lambda, \mathbf{k}, \mathrm{IWOC})$ is equivalent to the joint probability that $\overline{\mathrm{X}}_{\mathrm{i}}>0$ for $\mathrm{i}=1,2,3,4$. We are assuming that each voter's preferences are independent of other voters' preferences.

Some observations are made about the distribution of the $X_{i}$ 's that will be useful later. First, we note that the definitions of the $\mathrm{p}_{\mathrm{i}}$ 's under IWOC lead to the fact that $E\left(X_{i}\right)=0$ for $i=1,2,3,4$ where $E\left(X_{i}\right)$ denotes the expected value of $X_{i}$. The variance, $V\left(X_{i}\right)$, of $\mathrm{X}_{\mathrm{i}}$ is then given as $\mathrm{E}\left(\mathrm{X}_{\mathrm{i}}^{2}\right)$. With the definitions of the $\mathrm{X}_{\mathrm{i}}$ 's and IWOC we obtain

$$
\begin{gathered}
\mathrm{V}\left(\mathrm{X}_{1}\right)=\mathrm{V}\left(\mathrm{X}_{2}\right)=\frac{3 \mathrm{k}_{1}+2 \mathrm{k}_{2}}{3} \\
\mathrm{~V}\left(\mathrm{X}_{3}\right)=\mathrm{V}\left(\mathrm{X}_{4}\right)=\frac{2 \mathrm{k}_{1}}{3}\left(1-\lambda+\lambda^{2}\right)+\frac{\mathrm{k}_{2}}{12}\left(5-2 \lambda+2 \lambda^{2}\right)
\end{gathered}
$$

Covariance terms, $\operatorname{Cov}\left(X_{i}, X_{j}\right)$, are obtained as $E\left(X_{i} X_{j}\right)$ since $E\left(X_{i}\right)=0$ for $i=1,2,3,4$. With the definitions of the $X_{i}$ 's and the restrictions of IWOC on the $p_{i}$ 's, we obtain after algebraic reduction

$$
\begin{gathered}
\operatorname{Cov}\left(X_{1}, X_{2}\right)=\frac{1}{3}\left(k_{1}+k_{2}\right) \\
\operatorname{Cov}\left(X_{1}, X_{3}\right)=\operatorname{Cov}\left(X_{2}, X_{4}\right)=\frac{4 k_{1}+3 k_{2}}{6} \\
\operatorname{Cov}\left(X_{1}, X_{4}\right)=\operatorname{Cov}\left(X_{2}, X_{3}\right)=\frac{4 k_{1}+3 k_{2}}{12} \\
\operatorname{Cov}\left(X_{3}, X_{4}\right)=\frac{k_{1}}{3}\left(1-\lambda+\lambda^{2}\right)+\frac{k_{2}}{24}\left(5-2 \lambda+2 \lambda^{2}\right) .
\end{gathered}
$$

With these variance and covariance terms, the correlation matrix, $\mathrm{R}$, that is determined from the four $\mathrm{X}_{\mathrm{i}}$ variables is given by

$$
R=\left[\begin{array}{cccc}
1 & b & \rho & \rho / 2 \\
- & 1 & \rho / 2 & \rho \\
- & - & 1 & 1 / 2 \\
- & - & - & 1
\end{array}\right]
$$


where

$$
\begin{gathered}
b=\frac{\mathrm{k}_{1}+\mathrm{k}_{2}}{3 \mathrm{k}_{1}+2 \mathrm{k}_{2}}, \\
\rho=\frac{4 \mathrm{k}_{1}+3 \mathrm{k}_{2}}{\sqrt{\left(3 \mathrm{k}_{1}+2 \mathrm{k}_{2}\right) \mathrm{z}}}, \text { and } \\
\mathrm{z}=\left[8 \mathrm{k}_{1}\left(1-\lambda+\lambda^{2}\right)+\mathrm{k}_{2}\left(5-2 \lambda+2 \lambda^{2}\right)\right] .
\end{gathered}
$$

We return to our definition of $\mathbf{J}(\lambda, \mathbf{k}$, IWOC $)$ as the joint probability that $\overline{\mathrm{X}}_{\mathrm{i}}>0$ for $\mathrm{i}=1,2,3,4$. It follows directly for $\mathrm{n}$ voters, that $\mathrm{J}(\boldsymbol{\lambda}, \mathbf{k}$, IWOC $)$ is equivalent to the joint probability that $\bar{X}_{i} \sqrt{n}>0$ for $i=1,2,3,4$. In the limit that $n \rightarrow \infty$, the probability that any $\overline{\mathrm{X}}_{\mathrm{i}}$ takes on any specific value, such as zero goes to zero. Thus, $J(\lambda, \mathbf{k})$ is equivalent to the joint probability that $\bar{X}_{i} \sqrt{n} \geq 0$ for $i=1,2,3,4$. As a final step, we use the fact that $E\left(\bar{X}_{i}\right)=0$ to observe that $J(\lambda, \mathbf{k}$, IWOC $)$ is equivalent to the joint probability that $\bar{X}_{i} \sqrt{n} \geq E\left(\bar{X}_{i} \sqrt{n}\right)$ for $i=1,2,3,4$. This defines a positive orthant probability of the four-variate distribution on the $\bar{X}_{i} \sqrt{n}$ 's. In the limit as $n \rightarrow \infty$, the Central Limit Theorem requires this joint distribution to be multivariate normal with correlation matrix $\mathrm{R}$, as defined above.

Let $\Phi_{4}(\mathrm{R})$ denote the positive orthant probability for the four-variate normal distribution with correlation matrix R. Much is known about representations for $\Phi_{4}(\mathrm{R})$, depending upon the specific form of R [Johnson and Kotz (1972)]. The symmetry of IOWC with respect to candidates requires that $\mathrm{J}(\lambda, \mathbf{k}, \mathrm{IWOC})=3 \Phi_{4}(\mathrm{R})$. Given this representation, some observations can be made with regard to how $\mathrm{J}(\boldsymbol{\lambda}, \mathbf{k}, \mathrm{IWOC})$ changes as $\mathrm{k}_{1}$ and $\mathrm{k}_{2}$ change.

Theorem 1. $\mathrm{J}(\lambda, \mathbf{k}, \mathrm{IWOC})$ increases as $\mathrm{k}_{2}$ increases, and it decreases as $\mathrm{k}_{1}$ increases.

Proof: A result due to Slepian (1962) can be applied directly in this case to prove the first part of the statement. In particular, if some elements of $\mathrm{R}$ will increase, and none 
will decrease as $\mathrm{k}_{2}$ increases, $\Phi_{4}(\mathrm{R})$ will increase. From our definitions, it is sufficient to show that both $\rho$ and $b$ increase as $k_{2}$ increases. After taking derivatives, we find

$$
\begin{gathered}
\frac{\mathrm{db}}{\mathrm{dk}_{2}}=\frac{\mathrm{k}_{1}}{\left(3 \mathrm{k}_{1}+2 \mathrm{k}_{2}\right)^{2}} \\
\frac{\mathrm{d} \rho}{\mathrm{dk}_{2}}=\frac{4 \mathrm{k}_{1}^{2}\left(5-14 \lambda+14 \lambda^{2}\right)+\mathrm{k}_{1} \mathrm{k}_{2}\left(13-34 \lambda+34 \lambda^{2}\right)}{2\left[\left(3 \mathrm{k}_{1}+2 \mathrm{k}_{2}\right) \mathrm{z}\right]^{3 / 2}} .
\end{gathered}
$$

Both terms are strictly positive for all $\lambda \in[0,1]$, to prove the first part of the statement.

To prove the second part of the statement, we use the fact that if some elements of $\mathrm{R}$ will decrease, and none will increase as $\mathrm{k}_{1}$ increases; then $\Phi_{4}(\mathrm{R})$ will decrease. It is sufficient to show that both $\rho$ and $\mathrm{b}$ decrease as $\mathrm{k}_{1}$ increases. After taking derivatives, we find

$$
\begin{gathered}
\frac{\mathrm{db}}{\mathrm{dk}_{1}}=\frac{-\mathrm{k}_{2}}{\left(3 \mathrm{k}_{1}+2 \mathrm{k}_{2}\right)^{2}} \\
\frac{\mathrm{d} \rho}{\mathrm{d \textrm {k } _ { 1 }}}=\frac{-4 \mathrm{k}_{1} \mathrm{k}_{2}\left(5-14 \lambda+14 \lambda^{2}\right)-\mathrm{k}_{2}^{2}\left(13-34 \lambda+34 \lambda^{2}\right)}{2\left[\left(3 \mathrm{k}_{1}+2 \mathrm{k}_{2}\right) \mathrm{z}\right]^{3 / 2}} .
\end{gathered}
$$

Both terms are strictly negative for all $\lambda \in[0,1]$, to prove the second part of the statement.

\section{QED}

Following our earlier definitions, the Condorcet efficiency of Rule $\lambda$ as $n \rightarrow \infty$ under IOWC is given by $\mathrm{CE}(\lambda, \mathbf{k}, \mathrm{IWOC})$, with

$$
\mathrm{CE}(\lambda, \mathbf{k}, \mathrm{IWOC})=\frac{\mathrm{J}(\lambda, \mathbf{k}, \mathrm{IWOC})}{\mathrm{P}_{\mathrm{Con}}^{\infty}(\mathrm{IWOC})} .
$$

It would be very interesting to extend the results of Theorem 1 to consider the impact that changing $\mathrm{k}_{1}$ and $\mathrm{k}_{2}$ have on Condorcet efficiency of voting rules. However, both $\mathrm{J}(\lambda, \mathbf{k}$, IWOC $)$ and $\mathrm{P}_{\mathrm{Con}}^{\infty}(\mathrm{IWOC})$ increase as $\mathrm{k}_{2}$ increases, as a result of Theorem 1 and Lemma 1 respectively. Thus, it is not immediately obvious as to the effect that changing $\mathrm{k}_{2}$ has on their ratio. Similarly, both $\mathrm{J}(\lambda, \mathbf{k}$, IWOC $)$ and $\mathrm{P}_{\mathrm{Con}}^{\infty}(\mathrm{IWOC})$ are 
decreasing as $\mathrm{k}_{1}$ increases, so it is not obvious as to the effect that changing $\mathrm{k}_{1}$ has on their ratio.

Some interesting results do follow from the representation for $\operatorname{CE}(\lambda, \mathbf{k}, \mathrm{IWOC})$ for Rule $\lambda$, with $\lambda=1 / 2$, which is a special case that is known as Borda Rule.

Theorem 2. $\mathrm{CE}(\lambda, \mathbf{k}, \mathrm{IWOC})=\mathrm{CE}(1-\lambda, \mathbf{k}, \mathrm{IWOC})$ for all $\lambda \in[0,1]$, and $\mathrm{CE}(\lambda, \mathbf{k}, \mathrm{IWOC})$ increases as $\lambda$ increases from 0 to $1 / 2$, with $\mathrm{CE}(\lambda, \mathbf{k}, \mathrm{IWOC})$ being maximized at $\lambda=1 / 2$.

Proof: Since $\mathrm{P}_{\mathrm{Con}}^{\infty}(\mathrm{IWOC})$ is not a function of $\lambda$, the statement will be proved as long as it can be shown to be true for $\Phi_{4}(\mathrm{R})$. The first part of the statement is obviously true from the fact that $\mathrm{R}$ remains the same if $\lambda$ is replaced by $1-\lambda$ in the function $\mathrm{z}$ within the definition of $\rho$. The results from Slepian (1962) are then applied directly in this case, as in the proof of Theorem 1, to prove the second part of the statement. In particular, since some elements of $\mathrm{R}$ will increase, and none will decrease as $\rho$ increases; $\Phi_{4}(\mathrm{R})$ will increase as $\rho$ increases. From our definitions, $\rho$ will increase as $z$ decreases. The results then follows directly by examining the derivative of $\mathrm{z}$ with respect to $\lambda$.

\section{QED}

Based on this result, we see that Borda Rule is the WSR that maximizes Condorcet efficiency for three candidates under IWOC in the limit of voters for all $\mathbf{k}$. Gehrlein and Fishburn (1978) proved this result for the original case of IC, with $\mathrm{k}_{1}=1$. Van Newenhizen (1992) presents a similar result for a more general assumption regarding voter preference profiles when indifference is not allowed. Saari (1990) shows that Borda Rule also has many other interesting properties to support its use as a voting rule.

The results of Lemma 1 suggest that the special case of $\mathrm{k}_{1}=0$ and $\mathrm{k}_{2}>0$ are of special interest, since this results in $\mathrm{P}_{\mathrm{Con}}^{\infty}(\mathrm{IWOC})=1$, so that we are certain to have a Condorcet winner. The correlation matrix $\mathrm{R}$ takes on a special form, $\mathrm{R}^{*}$, in this situation, with 


$$
\mathrm{R}^{*}=\left[\begin{array}{cccc}
1 & 1 / 2 & \rho & \rho / 2 \\
- & 1 & \rho / 2 & \rho \\
- & - & 1 & 1 / 2 \\
- & - & - & 1
\end{array}\right]
$$

A correlation matrix of this particular form is of special interest since David and Mallows (1961) have obtained a closed form representation for $\Phi_{4}\left(\mathrm{R}^{*}\right)$ as

$$
\Phi_{4}\left(R^{*}\right)=\frac{1}{9}+\frac{1}{4 \pi^{2}}\left[\operatorname{Sin}^{-1}(\rho)+\operatorname{Sin}(\rho / 2)\right]\left[\operatorname{Sin}^{-1}(\rho)-\operatorname{Sin}^{-1}(\rho / 2)+\pi\right] .
$$

Let $\mathbf{k}^{*}$ define any $\mathbf{k}$ with $\mathrm{k}_{1}=0$ and $\mathrm{k}_{2}>0$. Given the discussion above, it follows that the Condorcet efficiency of Rule $\lambda$ in the limit of voters with three candidates is given by

$$
\operatorname{CE}\left(\lambda, \mathbf{k}^{*}, \mathrm{IWOC}\right)=\frac{1}{3}+\frac{3}{4 \pi^{2}}\left[\operatorname{Sin}^{-1}\left(\rho^{*}\right)+\operatorname{Sin}^{-1}\left(\rho^{*} / 2\right)\right]\left[\operatorname{Sin}^{-1}\left(\rho^{*}\right)-\operatorname{Sin}\left(\rho^{*} / 2\right)+\pi\right],
$$

where

$$
\rho^{*}=\frac{3}{\sqrt{2\left(5-2 \lambda+2 \lambda^{2}\right)}} .
$$

The results in Theorem 2 require that $\mathrm{CE}\left(\lambda, \mathbf{k}^{*}, \mathrm{IWOC}\right)=\mathrm{CE}\left(1-\lambda, \mathbf{k}^{*}, \mathrm{IWOC}\right)$ and that $\mathrm{CE}\left(\lambda, \mathbf{k}^{*}, \mathrm{IWOC}\right)$ increases as $\lambda$ increases for $\lambda \in[0,1 / 2]$. An interesting observation is that when we substitute $\lambda=1 / 2$, we get $\rho^{*}=1$, and

$$
\mathrm{CE}\left(1 / 2, \mathbf{k}^{*}, \mathrm{IWOC}\right)=1 \text {. }
$$

Thus, we find that when no voters have complete preference rankings, and some proportion of them have partial indifference, that there must be a Condorcet winner, and that Borda Rule must select it for three candidates as $n \rightarrow \infty$ under IWOC. From the perspective of maximizing Condorcet efficiency, there is no better situation than to be using Borda Rule to determine the winner, and to have all voters' preferences represented by partial indifference. Obviously, it is not possible to have greater Condorcet efficiency in any case with $\mathrm{k}_{1}>0$, where some proportion of voters have complete preference structures. This leads us to consider the impact that voters with complete preference structures will have on the Condorcet efficiency of an election. 
We begin by finding a much more tractable representation for $\operatorname{CE}(\lambda, \mathbf{k}, \mathrm{IWOC})$ than the one given above. In particular, we find a representation for $\Phi_{4}(\mathrm{R})$ that is a function of a single integral. Plackett (1954) develops a procedure for obtaining $\Phi_{4}(\mathrm{R})$ from $\Phi_{4}\left(\mathrm{R}^{*}\right)$ with the addition of a single integral for each correlation term for which $\mathrm{R}$ varies from $\mathrm{R}^{*}$. Since our previously defined $\mathrm{R}$ and $\mathrm{R}^{*}$ are different in only one entry, and we have a closed form representation for $\Phi_{4}\left(\mathrm{R}^{*}\right)$, it is possible to obtain an elegant form for $\Phi_{4}(\mathrm{R})$ that can be easily evaluated by quadrature.

The first step of Plackett's Reduction Procedure is to form the matrix $\mathrm{C}(\mathrm{t})$ with $C(t)=t R+(1-t) R^{*}$. C (t) has entries denoted by $c_{i, j}$. Then the matrix inverse of $C(t)$ is found with $H(t)=C(t)^{-1}$. This inverse matrix has entries denoted by $h_{i, j}$, and it is given by

$H(t)=\frac{1}{J}\left[\begin{array}{cccc}-4\left(\rho^{2}-1\right) & 2\left(\rho^{2}-2 a\right) & 4 \rho\left(\rho^{2}-1\right) & -2 \rho\left(\rho^{2}-2 a\right) \\ - & -4\left(\rho^{2}-1\right) & -2 \rho\left(\rho^{2}-2 a\right) & 4 \rho\left(\rho^{2}-1\right) \\ - & - & \frac{4}{3}\left(4 a \rho^{2}-4 a^{2}-5 \rho^{2}+4\right) & -\frac{4}{3}\left(5 a \rho^{2}-2 a^{2}-4 \rho^{2}+2\right) \\ - & - & - & \frac{4}{3}\left(4 a \rho^{2}-4 a^{2}-5 \rho^{2}+4\right)\end{array}\right]$,

where

$$
\begin{gathered}
\left.\mathrm{J}=\left(3 \rho^{2}-2 \mathrm{a}-2\right)\left(\rho^{2}+2 \mathrm{a}-1\right)\right), \quad \text { and } \\
\mathrm{a}=\frac{3 \mathrm{k}_{1}+2 \mathrm{k}_{2}-\mathrm{k}_{1} \mathrm{t}}{2\left(3 \mathrm{k}_{1}+2 \mathrm{k}_{2}\right)} .
\end{gathered}
$$

Using Plackett's Reduction Procedure

$$
\Phi_{4}(\mathrm{R})=\Phi_{4}(\mathrm{R} *)+\mathrm{I},
$$

where $\mathrm{I}$ is the integral term to account for the difference between $\mathrm{R}$ and $\mathrm{R}^{*}$. In particular, $\mathrm{R}$ and $\mathrm{R}^{*}$ are identical except for the difference $r_{1,2}=\mathrm{b}$ and $r_{1,2}^{*}=1 / 2$. Then we find $\mathrm{I}$ as

$$
\mathrm{I}=\frac{\mathrm{c}_{1,2}^{\prime}}{4 \pi^{2}} \int_{0}^{1}\left[\frac{1}{1-\mathrm{c}_{1,2}^{2}}\right]^{\frac{1}{2}} \operatorname{Cos}^{-1}\left(\frac{\mathrm{h}_{3,4}}{\sqrt{\mathrm{h}_{3,3} \mathrm{~h}_{4,4}}}\right) \mathrm{dt} .
$$

Where $\mathrm{c}^{\prime}{ }_{1,2}=\frac{\partial \mathrm{c}_{1,2}}{\partial \mathrm{t}}$. 
After substitution and significant algebraic reduction, we find

$$
\begin{gathered}
\Phi_{4}(\mathrm{R})=\frac{1}{9}+\frac{1}{4 \pi^{2}}\left[\operatorname{Sin}^{-1}(\rho)+\operatorname{Sin}(\rho / 2)\right]\left[\operatorname{Sin}^{-1}(\rho)-\operatorname{Sin}^{-1}(\rho / 2)+\pi\right] . \\
-\frac{\mathrm{k}_{1}}{4 \pi^{2}} \int_{0}^{1}\left[4\left(3 \mathrm{k}_{1}+2 \mathrm{k}_{2}\right)^{2}-\left(3 \mathrm{k}_{1}+2 \mathrm{k}_{2}-\mathrm{k}_{1} \mathrm{t}\right)^{2}\right]^{-\frac{1}{2}} \operatorname{Cos}^{-1}\left(\frac{3 \mathrm{k}_{1} \mathrm{t}\left(4 \mathrm{k}_{1}+3 \mathrm{k}_{2}\right)^{2}-\mathrm{g}}{2 \mathrm{~g}}\right) \mathrm{dt}
\end{gathered}
$$

where:

$$
\begin{gathered}
\mathrm{g}=4\left(3 \mathrm{k}_{1}+2 \mathrm{k}_{2}\right)^{2} \mathrm{z}-\left(3 \mathrm{k}_{1}+2 \mathrm{k}_{2}-\mathrm{k}_{1} \mathrm{t}\right)^{2} \mathrm{z}-\left(9 \mathrm{k}_{1}+6 \mathrm{k}_{2}+2 \mathrm{k}_{1} \mathrm{t}\right)\left(4 \mathrm{k}_{1}+3 \mathrm{k}_{2}\right)^{2}, \text { and } \\
\mathrm{z}=\left[8 \mathrm{k}_{1}\left(1-\lambda+\lambda^{2}\right)+\mathrm{k}_{2}\left(5-2 \lambda+2 \lambda^{2}\right)\right] .
\end{gathered}
$$

This representation for $\Phi_{4}(\mathrm{R})$ does not allow for direct integration to consider the impact on $\mathrm{CE}(\lambda, \mathbf{k}, \mathrm{IWOC})$ from changing $\mathrm{k}_{1}$ and $\mathrm{k}_{2}$. However, it does allow for direct calculation of values of $\mathrm{CE}(\lambda, \mathbf{k}, \mathrm{IWOC})$ by quadrature. A FORTRAN program was written to evaluate $\Phi_{4}(\mathrm{R})$ by numerical integration, to obtain estimates of $\operatorname{CE}(\lambda, \mathbf{k}$, IWOC $)$ for each $\lambda=0.00(.05) 0.50$ with every combination of $k_{1}=0.00(.10) 1.00$ and $\mathrm{k}_{2}=0.00(.10)\left(1-\mathrm{k}_{1}\right)$. Summaries of some computed values of $\mathrm{CE}(\lambda, \mathbf{k}, \mathrm{IWOC})$ are shown in Figure 1 for $\mathrm{k}_{1}=.1$, in Figure 2 for $\mathrm{k}_{1}=.3$, in Figure 3 for $\mathrm{k}_{1}=.5$ and in Figure 4 for $\mathrm{k}_{2}=.1$. Based on all computed results, we are led to the following conjecture that is true over the range of computed values. Given the wide range of values over which this conjecture is verified numerically, it seems quite unlikely that it is not generally true.

Conjecture 1. $\mathrm{CE}(\lambda, \mathbf{k}, \mathrm{IWOC})$ increases as $\mathrm{k}_{2}$ increases, and it decreases as $\mathrm{k}_{1}$ increases.

Thus, we reach the same conclusions for the Condorcet efficiency of voting rules as we did for the probability that a Condorcet winner exists under IWOC. That is, the existence of increased partial indifference will increase Condorcet efficiency, while the existence of an increased number of complete preference rankings will decrease Condorcet efficiency. As a result of Conjecture 1, IC (or any other situation with $\mathrm{k}_{2}=0$ and $k_{1}>0$ ) represents a worst case scenario for the evaluation of WSR's over the range of IWOC conditions. 


\section{The Effect of Forcing Preference Rankings}

It is interesting to consider what might happen if voters are forced to report complete preference rankings, rather than allowing them to state indifference between candidates. It is assumed that all indifference relations would be broken in a random fashion. Then a WSR would be applied to the reported complete ranking. Consider an example with partial indifference given by: $\mathrm{A} \sim \mathrm{B}, \mathrm{A} \succ \mathrm{C}$, and $\mathrm{B} \succ \mathrm{C}$. With random rankings on indifference, there are two possible rankings that could result from the forced ranking scenario: $\mathrm{A} \succ \mathrm{B} \succ \mathrm{C}$ and $\mathrm{B} \succ \mathrm{A} \succ \mathrm{C}$

For a given Rule $\lambda$, A would receive 1 point in the first case and $\lambda$ points in the second case. With both cases being equally likely, the expected number of points that are given to $\mathrm{A}$ is $(1+\lambda) / 2$, and the same is true for $\mathrm{B}$. Candidate $\mathrm{C}$ receives zero points in both cases. The WSR's that we defined for IWOC with partial indifference awards points to candidates in complete agreement with the expected number of points that would be awarded by the forced ranking scenario. It is straightforward to verify this result for other partial and complete indifference preference structures.

While the expected number of points that are awarded to candidates is identical under WSR's as defined for IWOC and the forced ranking scenario, we find very different results for the Condorcet efficiency of the two procedures.

Theorem 3. $\mathrm{CE}(\lambda, \mathbf{k}, \mathrm{IWOC})$ under forced ranking is identical to $\mathrm{CE}(\lambda, \mathbf{k}, \mathrm{IC})$.

Proof. With forced ranking under IWOC, the $\mathrm{X}_{\mathrm{i}}$ 's that are used in the development of correlation matrix $\mathrm{R}$ are based upon the six possible complete preference rankings. The probability that is associated with each ranking is then obtained from an accumulation over the different situations from which each complete ranking might be obtained. Arbitrarily consider the complete ranking $\mathrm{A} \succ \mathrm{B} \succ \mathrm{C}$ that was obtained by the forced ranking scenario. The first possibility, is that the voter had that particular complete preference ranking, which happens with probability $\mathrm{p}_{1}=\mathrm{k}_{1} / 6$. A second possibility is that $\mathrm{A} \succ \mathrm{B} \succ \mathrm{C}$ results from forced ranking on a preference structure with partial 
indifference. There are two possible partial indifference situations that can result in this particular forced ranking. With random ranking on indifference, the probability of obtaining $\mathrm{A} \succ \mathrm{B} \succ \mathrm{C}$ from the set of partial indifference preference structures is given by $1 / 2 p_{7}+1 / 2 p_{10}$, which equals $k_{2} / 6$ under IWOC. There are six equally likely forced preference rankings from the situation of complete indifference, so the probability that $\mathrm{A} \succ \mathrm{B} \succ \mathrm{C}$ results is $\mathrm{p}_{13} / 6=\mathrm{k}_{3} / 6$ under IWOC. The accumulated probability of obtaining the ranking $\mathrm{A} \succ \mathrm{B} \succ \mathrm{C}$ is then given by $\left(\mathrm{k}_{1}+\mathrm{k}_{2}+\mathrm{k}_{3}\right) / 6=1 / 6$, which is equivalent to IC. The same is true, by symmetry, for each of the other complete preference rankings that could result from forced preference.

QED

The results from Theorem 3 and Conjecture 1 lead us to the conclusion that the effect of forcing voters to report complete preference rankings, with indifferences being ranked randomly, reduces the Condorcet efficiency of all WSR's under IWOC. In fact, forced ranking results in a worst case scenario for the given WSR by obtaining the minimum possible Condorcet efficiency. 


\section{References}

de Condorcet , Marquis (1785). 'Essai sur l'Application de l'Analyse à la probabilité des Décisions Rendues à la Pluralité des Voix," (translated in The Political Theory of Condorcet, F. Sommerlad and I. McLean, Social Studies Working Paper 1/89, Oxford, 1989).

David, F. N. and C. L. Mallows (1961). “The variance of Spearman's rho in normal samples," Biometrika 48: 19-28.

Fishburn, P. C. and W. V. Gehrlein (1980). "The paradox of voting: Effects of individual indifference and intransitivity," Journal of Public Economics 14: 83-94.

Gehrlein, W.V. (1997). “Condorcet's Paradox and the Condorcet Efficiency of Voting Rules," Mathematica Japonica 45: 173-199.

Gehrlein, W.V. and P. C. Fishburn (1978). "Coincidence Probabilities for Simple Majority and Positional Voting Rules," Social Science Research 7: 272-283.

Guilbaud, G. T. (1952). "Les théories de l'intérêt général et le problème logique de l'agrégation," Economie Appliquée 5: 501-584

Inada, K. (1964). “A note on simple majority decision rule,” Econometrica 32: $525-531$

Jones, B., B. Radcliff, C. Taber and R. Timpone (1995). "Condorcet winners and the paradox of voting: Probability calculations for weak preference orders," American Political Science Review 89: 137-144.

Johnson, N.L. and S. Kotz (1972). Distributions in Statistics: Continuous Multivariate Distributions, Wiley, New York, NY.

Lepelley, D. and M. Martin (1998). “Condorcet's paradox for weak preference orderings," mimeograph.

Plackett , R. L. (1954). "A reduction formula for normal multivariate integrals," Biometrika 41, 351-360.

Saari, D. G. (1990). "The Borda dictionary," Social Choice and Welfare 7: 279317.

Slepian, D. (1962). “The one sided barrier problem for Gaussian Noise,” Bell Systems Technical Journal 41: 463-501. 
Van Deemen, A. (1999). "The probability of the paradox of voting for weak preference orderings," Social Choice and Welfare 16: 171-182.

Van Newenhizen, J. (1992). "The Borda method is most likely to respect the Condorcet principle," Economic Theory 2: 69-83. 


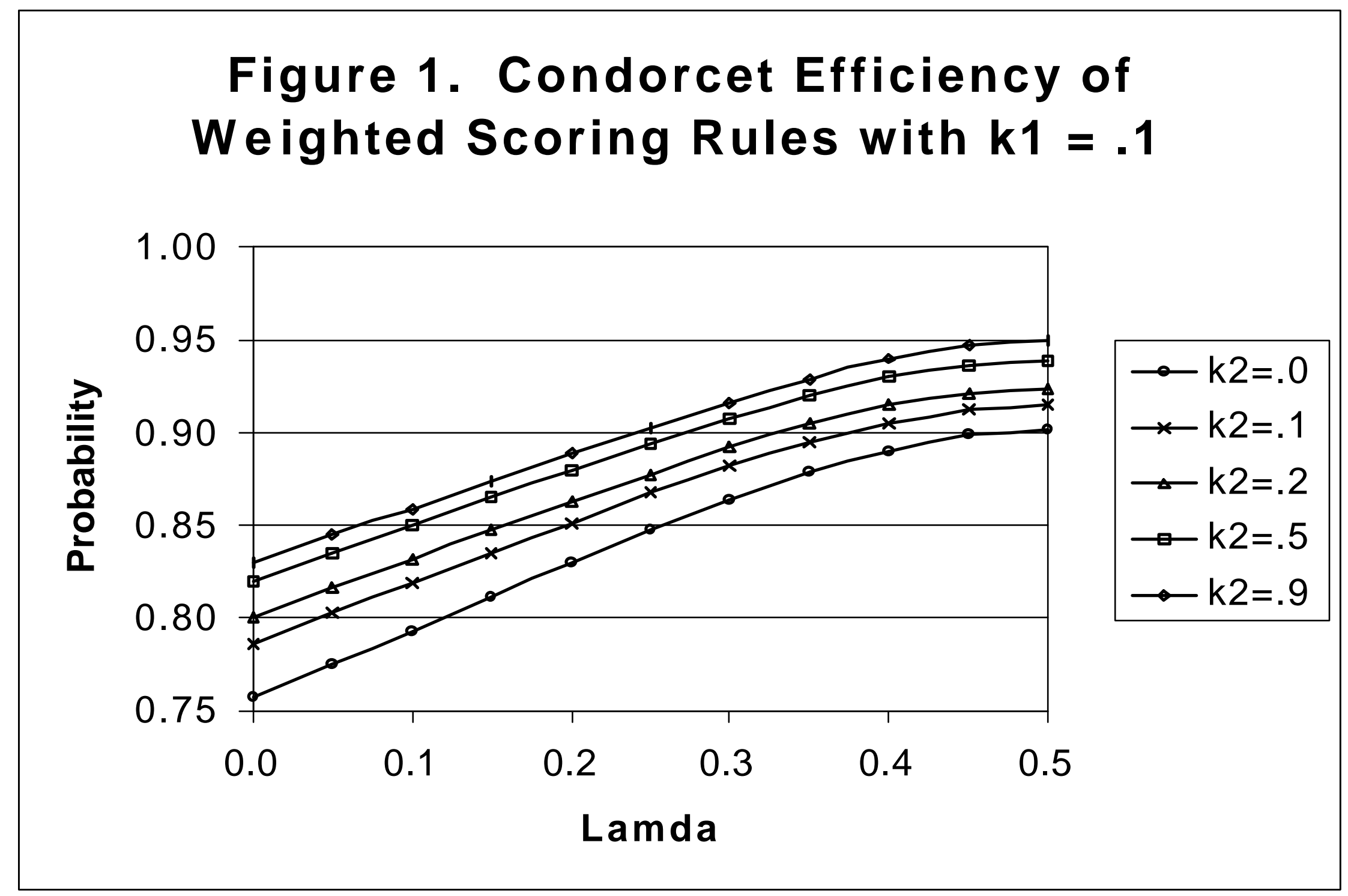




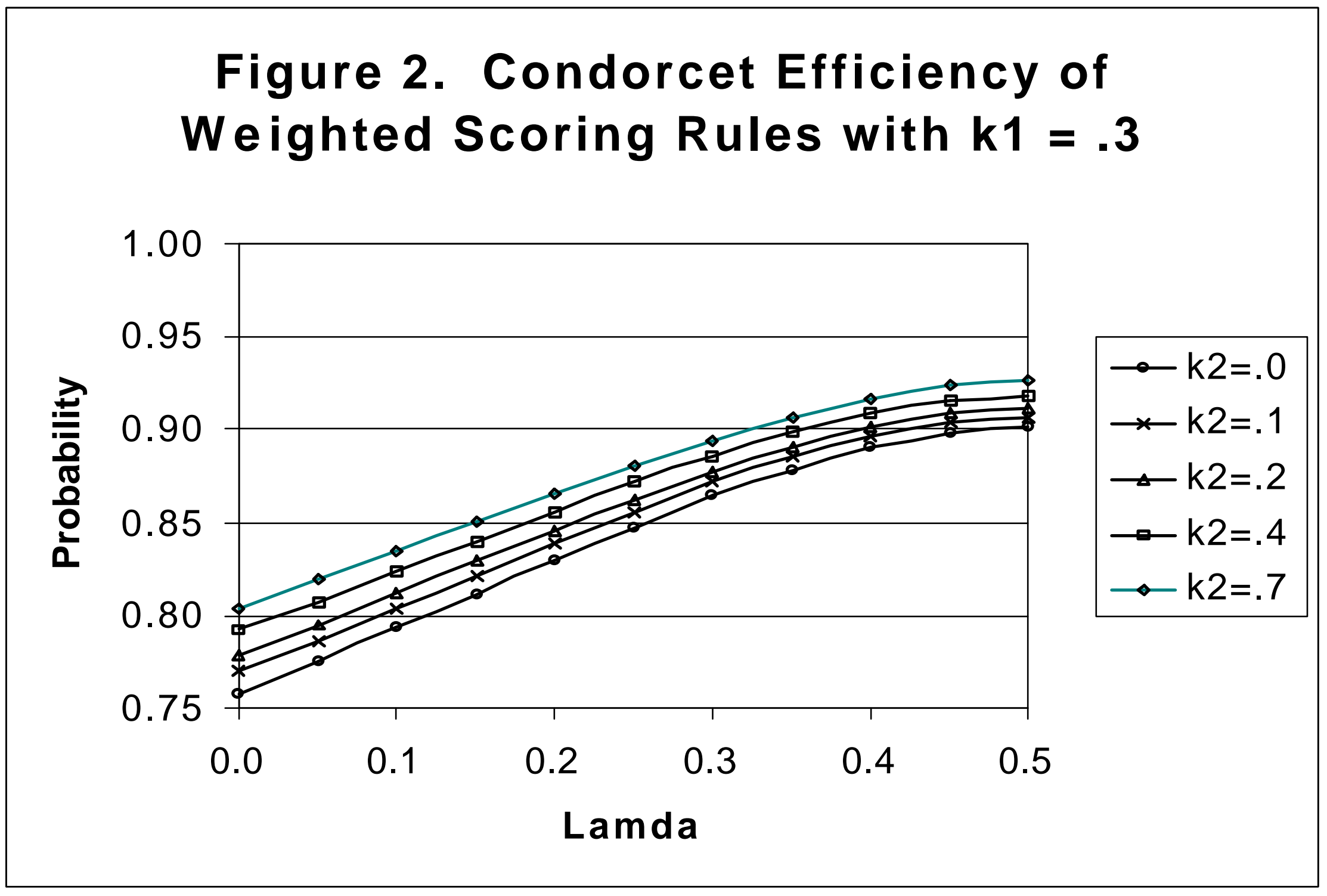




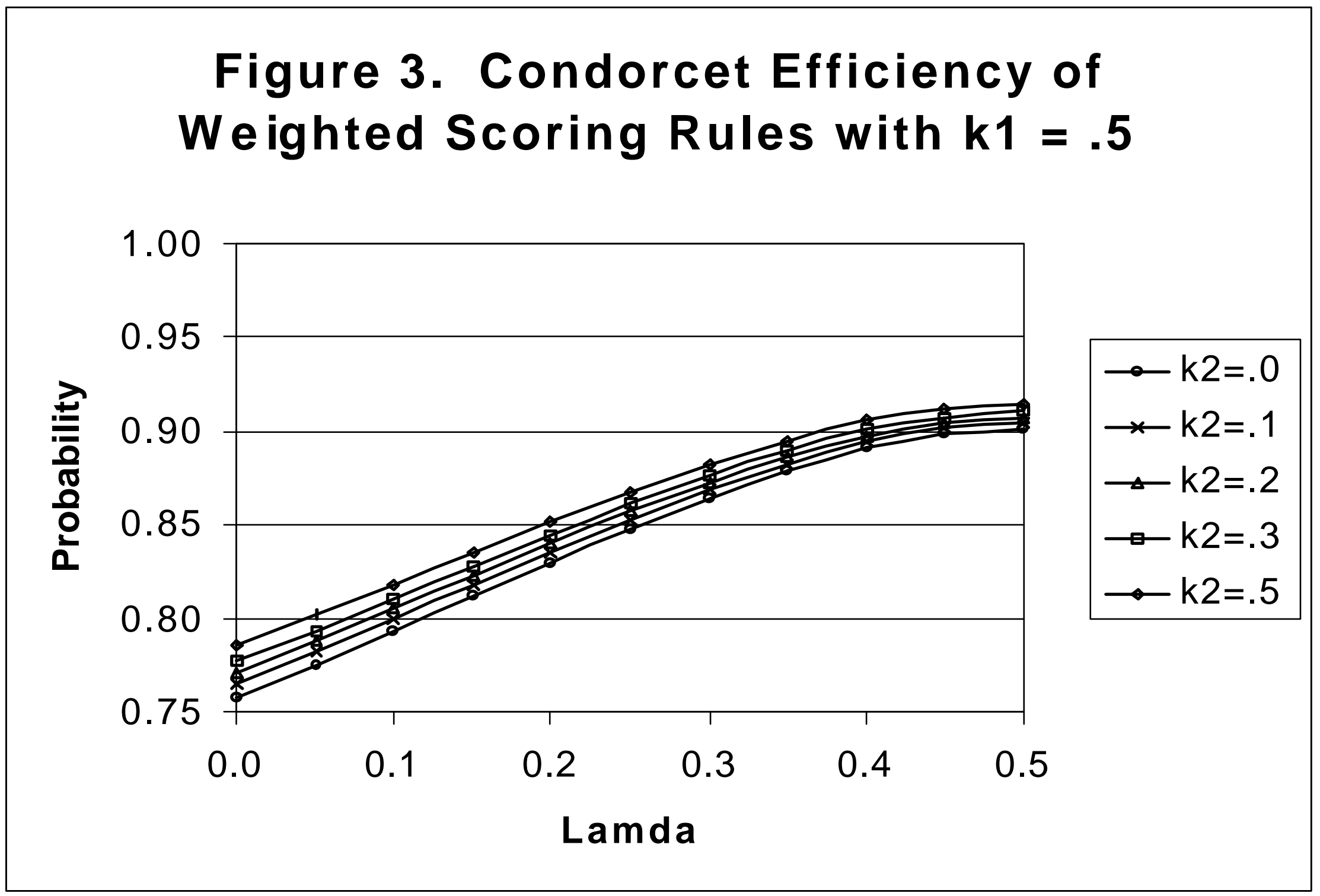




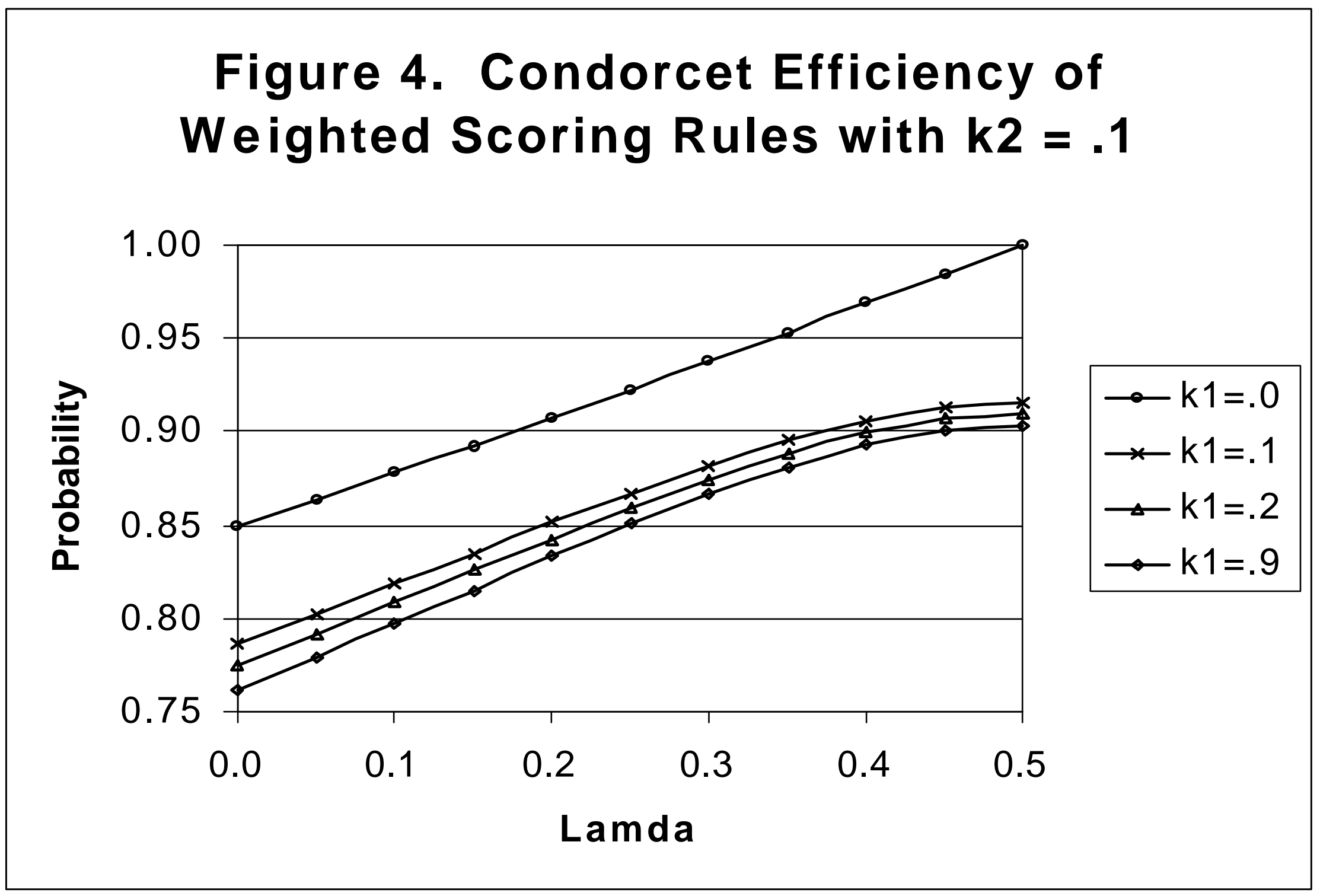

